\title{
Neurofibromatosis 2011: a report of the Children's Tumor Foundation Annual Meeting
}

\author{
Michel Kalamarides $\cdot$ Maria T. Acosta $\cdot$ Dusica Babovic-Vuksanovic $\cdot$ Olli Carpen $\cdot$ Karen Cichowski $\cdot$ \\ D. Gareth Evans · Filippo Giancotti - C. Oliver Hanemann • David Ingram • Alison C. Lloyd · Debra A. Mayes • \\ Ludwine Messiaen · Helen Morrison - Kathryn North - Roger Packer - Duojia Pan - Anat Stemmer-Rachamimov • \\ Meena Upadhyaya $\cdot$ David Viskochil $\cdot$ Margret R. Wallace $\cdot$ Kim Hunter-Schaedle $\cdot$ Nancy Ratner
}

Received: 24 August 2011/Revised: 21 September 2011/Accepted: 31 October 2011/Published online: 16 November 2011

(C) The Author(s) 2011. This article is published with open access at Springerlink.com

\begin{abstract}
The 2011 annual meeting of the Children's Tumor Foundation, the annual gathering of the neurofibromatosis (NF) research and clinical communities, was attended by 330 participants who discussed integration of new signaling pathways into NF research, the appreciation for NF mutations in sporadic cancers, and an expanding pre-clinical and clinical agenda. NF1, NF2, and schwannomatosis collectively affect approximately 100,000
\end{abstract}

\author{
M. Kalamarides \\ Hôpital Beaujon, Inserm U674, Universite Paris 7, Paris, France \\ M. T. Acosta $\cdot$ R. Packer \\ Children's National Medical Center, Washington, DC, USA \\ D. Babovic-Vuksanovic \\ Mayo Clinic, Rochester, MN, USA \\ O. Carpen \\ University of Turku, Turku, Finland \\ K. Cichowski \\ Harvard Medical School, \\ Brigham and Women's Hospital, Boston, MA, USA \\ D. Gareth Evans \\ MAHSC, University of Manchester, \\ St Mary's Hospital, Manchester, UK \\ F. Giancotti \\ Memorial Sloan-Kettering Cancer Center, New York, NY, USA \\ C. Oliver Hanemann \\ University of Plymouth, Plymouth, UK \\ D. Ingram \\ Indiana University, Indianapolis, IN, USA \\ A. C. Lloyd \\ MRC LMCB, University College London, London, UK
}

persons in US, and result from mutations in different genes. Benign tumors of NF1 (neurofibroma and optic pathway glioma) and NF2 (schwannoma, ependymoma, and meningioma) and schwannomatosis (schwannoma) can cause significant morbidity, and there are no proven drug treatments for any form of NF. Each disorder is associated with additional manifestations causing morbidity. The research presentations described in this review covered basic science, preclinical testing, and results from clinical

\section{A. Mayes}

Cincinnati Children's Hospital Medical Center,

Cincinnati, $\mathrm{OH}$, USA

L. Messiaen

University of Alabama at Birmingham, Birmingham, AL, USA

H. Morrison

Leibniz Institute for Age Research, Jena, Germany

K. North

The Children's Hospital at Westmead, Westmead, Australia

D. Pan

Johns Hopkins University, Baltimore, MD, USA

A. Stemmer-Rachamimov

Massachusetts General Hospital, Boston, MA, USA

M. Upadhyaya

Cardiff University, Wales, UK

D. Viskochil

University of Utah, Salt Lake City, UT, USA

M. R. Wallace

University of Florida, Gainesville, FL, USA

K. Hunter-Schaedle

Children's Tumor Foundation, New York, NY, USA 
trials, and demonstrate the remarkable strides being taken toward understanding of and progress toward treatments for these disorders based on the close interaction among scientists and clinicians.

\begin{tabular}{|c|c|}
\hline Abbreviations & \\
\hline ApoE & Apolipoprotein E \\
\hline AR12 & Akt inhibitor \\
\hline BMD & Bone density \\
\hline BRAF & $\begin{array}{l}\text { v-raf murine sarcoma viral oncogene } \\
\text { homolog B1 }\end{array}$ \\
\hline cAMP & Intracellular messenger \\
\hline CDKN2A & $\begin{array}{l}\text { Cyclin-dependent kinase inhibitor } 2 \mathrm{~A} \\
\text { (melanoma, p16, inhibits CDK4) }\end{array}$ \\
\hline CNPase & $\begin{array}{l}2^{\prime}, 3^{\prime} \text {-Cyclic nucleotide } 3^{\prime} \\
\text { phosphodiesterase }\end{array}$ \\
\hline CNS & Central nervous system \\
\hline CNV & Copy number variant \\
\hline $\mathrm{COG}$ & Children's Oncology Group \\
\hline CRL4-DCAF1 & E3 ubiquitin ligase \\
\hline CTEP-NCI & $\begin{array}{l}\text { Cancer Therapy Evaluation Program- } \\
\text { National Cancer Institute }\end{array}$ \\
\hline CTF & Children's Tumor Foundation \\
\hline dALK & $\begin{array}{l}\text { Drosophila homolog of the anaplastic } \\
\text { lymphoma kinase }\end{array}$ \\
\hline $\mathrm{DN}$ & Default network \\
\hline EMA & Epithelial membrane antigen \\
\hline ErbB2 & $\begin{array}{l}\text { v-erb-b2 erythroblastic leukemia viral } \\
\text { oncogene homolog } 2 \text {, neuro/glioblastoma } \\
\text { derived oncogene homolog (avian) }\end{array}$ \\
\hline EuroQOL & European quality of life \\
\hline F-actin & Filamentous actin \\
\hline $\mathrm{FC}$ & Functional brain connectivity \\
\hline GTPase & Guanosine triphosphatase \\
\hline GWA & Genome-wide association analysis \\
\hline HDAC & Histone deacetylase \\
\hline Hippo/Mst & $\begin{array}{l}\text { Drosophila and mammalian Ste } 20 \text { family } \\
\text { kinases }\end{array}$ \\
\hline HRasG12V & Activated form Ras \\
\hline HSW & Hippo/Salvador/Warts \\
\hline $\mathrm{IDH}$ & Isocitrate dehydrogenase \\
\hline INI1 & Integrase interactor 1 gene \\
\hline
\end{tabular}

N. Ratner $(\square)$

Division of Experimental Hematology and Cancer Biology,

Children's Hospital Medical Center, 3333 Burnet Ave.,

M.L.C. 7013, Cincinnati, OH 45229, USA

e-mail: Nancy.Ratner@cchmc.org

M. Kalamarides $(\square)$

Hôpital Beaujon, Neurochirurgie, 100 bd General Leclerc,

92110 Clichy, France

e-mail: michel.kalamarides@bjn.aphp.fr
Jak2/Stat5

Lats

LRES

MADM

MDM2

MEK

MGH

MPNST

mTOR

$\mathrm{NF}$

Nfl fl/fl

MRI T2

NFTI-QOL

NSC

OPC

p53/NF1

PDGFR

PET

PGDS

Plp-CreERT

P-MEK

PN

PRC

PTEN

Rac-Pak

Raf

Ras

Ras-MAPK

RB

RCAS/tv

SB

SF-36

SNP

SPRED

SUZ12

TEAD

TI

TP53

VEGF

VS

WHO

YAP1
Janus kinase 2 (a protein tyrosine kinase)/ signal transducer and activator of transcription 5

Large tumor suppressor homolog 1 gene

Long range epigenetic silencing

Mosaic analysis with double markers

Negative regulator of p53

MAP kinase-ERK kinase

Massachusetts General Hospital

Malignant peripheral nerve sheath tumor

FK506 binding protein 12-rapamycin associated protein 1

Neurofibromatosis

Neurofibromatosis 1 flox/flox

T2-weighted magnetic resonance image

Nursing facility transition initiative-quality of life

Neural stem cells

Oligodendrocyte precursor cell

Mutant p53 and NF1 genes

Platelet-derived growth factor receptor

Positron emission tomography

Prostaglandin D2 synthase

Inducible proteolipid protein cre

recombinase

Phosphorylated MAP kinase kinase

Plexiform neurofibroma

Polycomb repressive complex

Phosphatase and tensin homolog

Signaling downstream of the Rac small GTPase

v-raf-1 murine leukemia viral oncogene homolog 1

Monomeric GTPases

Signaling downstream of Ras

Retinoblastoma

Replication-competent ASLV long terminal repeat (LTR) with a splice acceptor/tv-a

gene.mouse modeling system

Sleeping beauty

Health survey

Single nucleotide polymorphism

Sprouty-related protein with EVH-1 domain

Suppressor of zeste 12 homolog (Drosophila)

DNA-binding partner of the YAP

coactivator

Transcriptional imbalances

Tumor protein p53

Vascular endothelial growth factor

Vestibular schwannoma

World Health Organization

Yes-associated protein 1, $65 \mathrm{kDa}$ 
Introduction to the meeting and the neurofibromatoses

The 2011 annual meeting of the Children's Tumor Foundation (CTF) NF Conference took place at the Snow King Resort, Jackson Hole, Wyoming, June 11th-14th, 2011. The CTF meeting is the annual gathering of the neurofibromatosis (NF) research and clinical communities. Since its inception in 1985, this conference has played a key role as a forum for fostering collaboration, consensus building, and advances in translational research. Meeting attendance has grown from 140 attendees in 2005 to over 300 attendees in 2011, driven by integration of new signaling pathways into NF research, the appreciation for NF pathways in sporadic diseases, and an expanding pre-clinical and clinical agenda component as the NF field progresses bench to bedside.

Neurofibromatosis is a group of inherited genetic disorders-NF1, NF2, and schwannomatosis-that together affect approximately 100,000 persons in US (http://www. ctf.org/Living-with-NF/learn-about-nf.html). NF1 is the most common, affecting 1:3,000 births; NF2 affects 1:25,000 births. NF1 and NF2 are autosomal dominant disorders: $50 \%$ of new cases arise from spontaneous mutations with no family history. Schwannomatosis affects an estimated 1:40,000 births. NF1, NF2 and schwannomatosis each predispose affected individuals to develop Schwann cell tumors. The disorders arise from mutations in different genes, each of which plays a key role in regulating Schwann cell function. The NFI gene on human chromosome 17 encodes an intracellular signaling molecule that functions as a GTPase activating protein for Ras proteins. In contrast, the NF2 gene on human chromosome 22 encodes a cytoskeletal-membrane linking protein. Understanding of Schwannomatosis is progressing since a candidate gene INII was identified.

Even the benign tumors of NF1 (neurofibromas and optic pathway gliomas) and NF2 (schwannoma, ependymoma, and meningioma) and schwannomatosis (schwannoma) can cause significant morbidity and even mortality. This is because of continuous growth and frequent surgical inaccessibility. In addition, malignancy and bone abnormalities, pain, vascular abnormalities, and in $60 \%$ of cases, learning disabilities complicate study of NF1. Pain is a debilitating consequence of schwannomatosis. There are as yet no proven drug treatments for any form of NF, but over the past several years multiple clinical trials have been initiated.

\section{Meeting report: keynote speakers}

Charles Eberhart (Johns Hopkins University School of Medicine) provided the opening keynote presentation. He focused on emerging links between NF pathways and sporadic gliomas. He reviewed reports identifying NF1 gene mutations in approximately $15 \%$ of sporadic human high grade glioma [4]. He then discussed the key role of BRAF signaling in sporadic pilocytic astrocytoma; most sporadic tumors have genetic alterations in BRAF, and $80 \%$ of non-NF1 pilocytic tumors show P-MEK staining, indicating activation of the same pathway affected in NF1associated pilocytic astrocytoma [2]. He emphasized the need to correlate genotype with outcome and tumor grade, and presented new work linking BRAF activity to oncogene-induced senescence in pilocytic astrocytoma, a process which may explain the sometimes indolent growth of optic gliomas in NF1 patients [19]. He also showed that a protein functioning downstream of NF2 (YAP1) is expressed in human neural stem cells, is associated with aggressive glioblastoma biology in patients, and that suppressing YAP1 levels in tumor cells slows their growth [16].

Stan Nelson (University of California, Los Angeles) described a powerful new strategy to identify novel Mendelian disease genes using whole exome sequencing. This approach reliably searches about $90 \%$ of the known protein coding sequence for all mutation types including single base substitutions, small indels, single and multiple exon deletions and duplications. His group and many others are identifying the cause of rare Mendelian diseases at a rapid pace, and the team at UCLA is using efficient and cost-effective whole exome sequencing to more rapidly diagnose individuals with genetic diseases. This approach has great promise for defining the spectrum of DNA variants that contribute to rare and common diseases and that may modify disease processes such as NF.

David Threadgill (North Carolina State University) gave the keynote presentation on Day 3 of the Conference. Dr. Threadgill leads a large international effort to exploit a new mouse genetic resource supporting the integration of genetics into systems biological analyses at the whole animal level. These efforts are based upon the Collaborative Cross, a set of unique recombinant inbred populations of mice that have randomly assorted the genetic polymorphisms present in eight founder inbred strains. $\mathrm{He}$ discussed his progress in identification and functional characterization of genes influencing susceptibility to diseases, and how researchers might use the Collaborative Cross to identify cancer modifier genes. Specifically, the $\mathrm{NF}$ community may consider using the Collaborative Cross to identify modifier genes relevant in particular to the most prevalent form of NF, NF1, because modifier genes are implicated in NF1 manifestations including numbers of dermal neurofibromas. 


\section{Update: neurofibromatosis type 1}

\section{The Brain in NF1}

NF1 patients are predisposed to CNS manifestations, mainly cognitive deficits, hyperintensities that appear on MRI imaging, and low grade pilocytic astrocytomas. NF1 mutations are also common in high grade glioma in the general population. Modeling of these phenotypes, understanding underlying mechanisms, and ongoing therapeutic trials were discussed.

Kathryn North (Children's Hospital Westmead, Australia) overviewed cognitive deficits and academic learning difficulties, the most common neurological disability in patients with NF1. She found that developmental deficits can be identified clinically in patients as young as 2 years old [15]. Some cognitive deficits may be associated with developmental or structural brain anomalies and are therefore unlikely to be reversible; these include increased grey matter volume and increased cross sectional area of the corpus callosum which are associated with lower IQ and learning deficits [18], and NF1 MRI T2-hyperintensities (non-enhancing bright areas on T2 images of unknown etiology) in the thalamus that are associated with reduced IQ [9]. Other cognitive deficits may be reversible and amenable to pharmacological therapy. Dr. North also overviewed published studies that used $N f 1$ mouse models. She described studies from the Silva group reversing defects in hippocampal based learning with Lovastin and noted that another mouse model, and humans, with abnormal ras activation due to mutations in SPREDI (Legius syndrome) also have impaired hippocampal based learning [5]. She described another NfI mouse model showing abnormal dopamine homeostasis underlying attention deficits that could be reversed by treatment with methylphenidate, suggesting a defect in brain catecholamine homeostasis [3]. These animal studies provide a strong imperative for pursuing definitive trials of stimulant medication in children with NF1.

Maria T. Acosta (Children's National Medical Center) found that lovastatin normalizes the brain spontaneous low-frequency fluctuations in children with NF1 [1]. She presented an initial examination of functional brain connectivity (FC) in Default Network (DN) architecture in $7 / 24$ patients treated with lovastatin as a part of a phase 1 study testing lovastatin as treatment for cognitive deficits in children with NF1 at the Gilbert Neurofibromatosis Institute. Lovastatin administration in this small sample appeared to normalize anterior-posterior and local functional connectivity within the DN. The pattern of results is consistent with normalization of developmental processes, and with apparent benefits in a mouse model. Although interpretation is necessarily tentative because of the sample size, these results suggest that continued examination of the potentially beneficial effects of lovastatin in NF1 is warranted.

The mechanisms underlying the cognitive changes in NF1 remain incompletely understood. Debra Mayes (Cincinnati Children's Hospital Medical Center) described her studies of how loss of $\mathrm{NfI}$ affects oligodendrocytes, the myelinating cell of the brain. She examined the optic nerve and corpus callosum white matter tracts in animal models. Phenotypes of $N f 1$ heterozygous mice, tamoxifen-induced NfI loss in mature oligodendrocytes (Plp-CreERT), and a new transgenic model in which the CNPase promoter drives expression of HRasG12V were presented. Expression HRasGV12 and loss of Nf1 (PLPCre;NfIfl+ \& PLPCre; Nflflffl) in oligo-lineage cells displayed similar defects in myelin, brain vasculature and axonal size, in the optic nerves and corpus callosum. These data support the idea that oligodendrocytes, as well as neurons and astrocytes in the CNS might contribute to patient deficits in NF1 disease.

A workshop on gliomas in NF highlighted problems in clinical management and diagnosis that relate to gliomas in the NF population. Anat Stemmer-Rachamimov (Massachusetts General Hospital) reviewed the World Health Organization (WHO) classification of gliomas. In the WHO classification scheme tumors are classified by type (as per type or cell or origin) and then assigned a histological grade. In NF1 patients, the predominant problem is that of grading of astrocytomas; specifically distinguishing between pilocytic astrocytomas (WHO grade I) from diffuse astrocytomas (WHO grades II, III and IV). The classic pilocytic astrocytomas are non-infiltrating, and have unique histological features including piloid cells, myxoid background, Rosenthal fibers and eosinophilic granular bodies. Pilocytic astrocytomas have excellent prognosis. Diffuse astrocytomas are divided into grades II, III and IV based on the degree of cellular density, cytological atypia, mitotic activity, necrosis and microvascular proliferation. Diffuse astrocytomas progress over time and higher grades (III, IV) require treatment with radiation and chemotherapy. The distinction between pilocytic astrocytomas and diffuse astrocytomas in NF1 patients may be difficult as some tumors present histological features that are intermediate.

David Gutmann (Washington University, St Louis) reviewed the clinical features of NF1-associated astrocytomas. The overwhelming majority of CNS tumors in NF1 are optic pathway pilocytic astrocytomas (WHO Grade I). However, diffuse low grade and high grade astrocytomas also occur. These tumors often present a diagnostic and treatment challenges as their radiological and pathological features are different from sporadic tumors and there are no correlational large studies to provide guidance for clinical management and treatment. 
Joann Ater (MD Anderson Cancer Center) presented data regarding treatment of progressive hypothalamic/optic pathway gliomas in children with NF1. The Children's Oncology Group (COG) evaluated tumor response in 127 children with or without NF1, and progressive hypothalamic/optic pathway gliomas treated on protocol COG A9952. Children with NF1 tolerated carboplatin and vincristine well and had tumor response rate and EFS that were superior to children without NF1. Second malignant neoplasms occurred only in patients who had progression and received secondary treatment with temozolomide.

Josh Rubin (Washington University St Louis) outlined a novel set of findings regarding the cAMP pathway in low grade Nfl-related brain tumors. Gliomagenesis can be induced by low levels of cAMP in a genetically engineered mouse model of NF1. However, tumor formation displays a significant sex-dependent disparity resulting in disease in $100 \%$ of male, but only $20 \%$ of female mice. The molecular basis for the difference in tumor rates in male and female mice appears to involve cell-intrinsic differences in cAMP regulation. These exciting studies may provide insight into why astrocytoma is more common in males than females and whether targeting the cAMP pathway in astrocytoma treatment will have equal value for males and females.

Anat Stemmer-Rachamimov (with Cathy Nutt) reviewed the literature on genomic anomalies associated with sporadic gliomas. Genes often altered in sporadic low grade astrocytomas include IDH1, IDH2, TP53 and PDGFR while EGFR, CDKN2A, chromosome 10 loss, $P T E N$ and $M D M 2$ alterations are seen in high grade gliomas. Recent large scale studies using gene expression profiling have identified four subclasses of glioblastomas based on transcriptional signatures: proneural, neural, mesenchymal and classic [21]. The mesenchymal subtype has been found to be associated with NF1 mutations. Other mutations found in the mesenchymal subgroup included PTEN, TP53, and RB1. This suggests that NF1 mutations aggregate with specific other mutations and these may affect prognosis and response to specific treatment. A large correlative study in which histology, molecular features and clinical outcome of gliomas in NF1 will be integrated would provide valuable information as to clinical management and treatment of these tumors, and identify prognostic indicators.

Hui Zong (University of Oregon) used his elegant mouse genetic mosaic system MADM (Mosaic Analysis with Double Markers) to identify glioma cell-of-origin by analyzing aberrations in individual brain cell lineages prior to the glioma formation. After initiating p53/NF1 mutations sporadically in neural stem cells (NSCs), they analyzed mutant NSCs and their progeny at pre-malignant stages. Their data pointed to a non-NSC cell type, the OPC cell, as the cell-of-origin for glioma [13]. Further transcriptome analysis and genetic evidences confirmed the essential role of OPCs in gliomagenesis. Their findings provide valuable guidance for cell-type specific treatment for malignant glioma.

The peripheral nervous system in NF1

NF1 patients are predisposed to benign Schwann cell tumors called neurofibromas, and to malignant peripheral nerve sheath tumors, an aggressive sarcoma which is a leading cause of death in NF1 adults. The discussion centered continuing improvement of mouse models, identification of mechanisms of neurofibroma formation, preclinical therapeutics in existing mouse models, and ongoing clinical trials.

Alison Lloyd (University College London, UK) reported on two novel mouse models to study neurofibroma formation. The lab has previously shown in in vitro studies that activation of the ERK signaling pathway was sufficient to drive myelinating Schwann cells back to the progenitorlike cells found in nerves following an injury and which resemble the Schwann cells found in neurofibromas. They constructed a transgenic mouse in which ERK signaling could be reversibly induced following tamoxifen injections. These studies confirmed that this pathway was sufficient to drive this switch in cell-state, and identified that signals from the Schwann cells were responsible for inducing the inflammatory response seen following injury and during tumor formation. The links to injury were confirmed in a second mouse-model in which neurofibromas developed from adult myelinating Schwann cells when NF1 loss was induced in concert with an injury.

The link between inflammation and neurofibroma development was further explored in the talk by Yuan Zhu (University of Michigan). He reported a detailed temporal analysis of tumor development in his Nf1 mouse model. He showed that $N f 1^{-/-}$Schwann cells mostly differentiate normally except there are abnormalities in Remak-bundle formation which resolved as the animals aged. However, at these later ages, non-myelinating Schwann cells appeared to start to dissociate from axons concomitant with mast cell infiltration. These later defects were associated with increased ERK and mTOR signaling. Treatment with Rapamycin blocked most of the Schwann cell dissociation, indicating a potential treatment to block tumor formation at an early stage.

Nancy Ratner (Cincinnati Children's Hospital Medical Center) emphasized that specific Ras signaling pathways that mediate neurofibroma and MPNST pathology remain unknown. She described cross-species transcriptome analysis to identify molecular pathways altered in NF1 tumors and guide molecular-targeted therapeutic strategies. The 
analysis identified global negative feedback of Ras-MAPK signaling was prominent in human and mouse. Inhibiting this pathway with a MEK inhibitor diminished human MPNST cell growth in xenografts and decreased neurofibroma volume in $85 \%$ of neurofibromas in a mouse model [22], providing strong rationale for testing MEK inhibitors in neurofibroma and MPNST human clinical trials.

Wade Clapp (Indiana University School of Medicine) described his efforts to develop novel experimental therapeutics for plexiform neurofibromas. He previously reported that a multi- receptor tyrosine kinase inhibitor (imatinib mesylate), a drug that targets c-kit, PDGF, and $\mathrm{c}$-abl, reduces plexiform neurofibromas in a mouse model [23]. He has screened ten drugs that are in various phases of clinical development of which two passed both short term and long term treatment objectives that included reducing the size of the tumors by $50 \%$ and reducing the absolute number of tumors by $25 \%$ or more. His current work is aimed at moving these drugs forward into phase 2 clinical trials.

Brigitte Widemann (National Cancer Institute) described previous human trials for plexiform neurofibroma, utilizing agents such as farnesyl-transferase inhibitors, antifibrotic drugs, and immunomodulary agents (interferon) that have been completed with mixed and somewhat disappointing results; these trials were based on some preclinical testing, but not detailed pre-clinical mouse modeling. The interactions between the human and mouse trials have become a two way road, informing both types of studies. The next generation of human clinical trials for plexiform neurofibromas will be heavily based on preclinical modeling. Imatinib (NCT01140360) and sorafenib (NCT00727233) are two trials which have been recently undertaken based on mouse modeling, but these studies point out the complexity of the transition from mouse models to human care.

Dusica Babovic-Vuksanovic (Mayo Clinic) provided an update on results from a CTEP-NCI multi-center phase 2 trial of cediranib for NF1 plexiform and/or paraspinal neurofibromas (NCT00326872). The study enrolled 26 adult patients who were treated with oral cediranib at $30 \mathrm{mg} /$ day over 26 cycles. The primary endpoint of therapy was a volume change of target tumors, based on 3-D MRI, measured by two independent blinded evaluators. A secondary endpoint was symptomatic improvement based on the Brief Pain Inventory. Four patients responded with decrease in tumor volume of $>20 \%$ and no radiological progression of disease was noted in any of evaluable patients. Brief Pain Index analysis showed reduction in pain or a reduction in the interference caused by pain in treated patients. Results are encouraging but more data is needed to assess efficacy of cediranib in patients with NF1.
Kent Robertson (Indiana University School of Medicine) presented data from a pilot phase 2 study of imatinib in NF1 patients with plexiform neurofibromas (PN) (NCT01140360). The primary endpoint was PN size by MRI cross sectional area and secondary endpoints included symptomatic improvement, expression of 15 plasma cytokines, and safety/toxicity of imatinib. Of 24 evaluable patients, $15(62 \%)$ responded with tumor reduction of one or more PN's; 9 (38\%) were non-responsive (stable or progressive tumors only). Out of 73 tumors scored, 22 (30\%) showed reduction while $22(30 \%)$ showed progression, and 29 tumors remained stable (40\%). Symptom improvement was reported by 6 of $15(40 \%)$ patients with responsive disease and 1 of $9(11 \%)$ of patients with nonresponsive tumors following 6 months of oral imatinib $(p=0.19)$. A panel of 15 plasma cytokines after 6 months of therapy showed greater than twofold increase in levels in $24 \%$ of cytokines measured in patients with non-responsive disease as compared to $2 \%$ in patients with responsive tumors $(p<0.001)$. Thus, a subset of NF1 patients with PN's treated with imatinib had reductions in tumor size associated with symptom improvement and cytokine expression correlated with tumor response.

Fawn Leigh (Massachusetts General Hospital/Harvard Medical School) presented data related to efforts to identify genetic modifiers of cutaneous neurofibroma tumor burden in NF1. The MGH group carried out a genome-wide association analysis (GWA) in 300 subjects with extreme burden of cutaneous tumors, using the Affymetrix GeneChip 6.0 platform, providing 909, 622 single nucleotide polymorphism (SNP) markers. While their preliminary data indicate that there is no single common modifier responsible for the variation in tumor burden, genotyping in an additional cohort of extreme subjects to confirm or refute its modifier status is ongoing. Copy number variant (CNV) analysis in their initial cohort is also in process. Importantly, the authors solicit collaboration from all NF Clinics interested in a collaborative community-wide effort to address the cooperative identification of genetic modifiers of NF1.

David Largaespada (University of Minnesota)'s laboratory uses insertional mutagenesis for cancer gene discovery in the mouse. By crossing mice harboring mutagenic Sleeping Beauty (SB) transposons to mice expressing SB transposase in specific cell-types they are able to identify novel driver genes for tumor formation in specific tissues. Using this approach to target Schwann cells, Largaespada reported the formation of a large number of SB-accelerated neurofibromas and malignant peripheral nerve sheath tumors. Analysis of the SB transposon insertion sites resulted in the identification of Nfl and Pten genes as recurrent insertional sites as well as a large number of other genes not previously identified as having a role in Schwann 
cell tumor formation. Interestingly, Pten and NfI inactivation often occurred in the same tumors arguing that NF1 loss may not be sufficient to achieve the levels of PI3kinase signaling required for tumor formation.

Luis F. Parada (University of Texas Southwestern Medical Center) presented exciting results from a screen for novel drugs to treat glioblastomas and MPNSTs for which current therapeutics are currently ineffective. He took the novel approach of isolating cancer stem cells from glioblastomas and MPNSTs from his mouse-tumor models. Growing these cells under more physiological conditions, he pooled cells from multiple tumors for each tumor type, to provide a target for a screen that should hit multiple independent tumors. He then screened a $200 \mathrm{~K}$ chemical library for small molecule drugs that differentially killed tumor cells versus normal cells. Interestingly, in this assay, a number of drugs that have been used therapeutically but with little success were ineffective. These screens have resulted in the identification of a number of new potential drugs that function at low concentrations and with some specificity, and thus are exciting candidates for future therapeutics.

Eduard Serra (Institut de Medicina Predictiva I Personalitzada del Cancer, Barcelona) correlated transcriptional profiles generated by the NF1 Microarray Consortium with SNP data from neurofibromas and MPNSTs. His group identified clusters of up-regulated or down-regulated genes that mapped in specific genomic regions (termed Transcriptional Imbalances, TIs), present in MPNSTs and not neurofibromas. Many TIs correlated with somatic copy number alterations (gains and losses of genetic material), with the best correlation with genomic gains rather than losses. Dr. Serra's group suggested that long range epigenetic silencing (LRES) could be the mechanism responsible for the generation of TIs representing clusters of down-regulated genes that were not explained by losses of genetic material, since there was a good overlapping between the precise chromosomal position of these TIs and LRES previously identified in prostate cancer.

Karen Cichowski (Brigham and Women's Hospital, Harvard Medical School) reviewed the finding that inhibition of the mTOR pathway alone is insufficient to cause MPNST cell death, and proposed a more comprehensive, cytotoxic approach. She described a new potential combination therapy for these tumors [6]. Specifically, she showed that agents that enhance proteotoxic stress, including HSP90 inhibitors, induce rapid tumor regression in an aggressive genetically engineered mouse cancer model, but only when combined with the mTOR inhibitor, rapamycin. Her lab found that these agents synergize by promoting irresolvable proteotoxic stress and inducing catastrophic endoplasmic reticulum and mitochondrial damage. Moreover, Dr. Cichowski found that this combination therapy is also effective in a genetically engineered mouse model of K-Ras mutant lung cancer. These studies have defined a specific and promising drug combination that can be assessed in patients with MPNSTs.

Dina Lev (MD Anderson) is studying the impact of drug-induced autophagy in cancer. While several drugs promote tumor cell death via a possible autophagic mechanism and/or apoptosis, such drugs may also promote the unintentional survival of cancer cells, indicating a likely role for drug-induced autophagy as a component of tumor chemoresistance. Her group has demonstrated that blockade of the PI3K/mTOR pathway and inhibtion of HDAC induce autophagy in human MPNST cells, both in vitro and in xenografts [14]. Furthermore, such genetic and chemical blockade of drug-induced autophagy significantly enhanced therapeutic apoptotic MPNST cell death, a clear indication that drug-induced autophagy represents a survival mechanism in MPNST that may identify a new focus of therapy development to prevent MPNSTs from becoming resistant to chemotherapy.

\section{NF1 manifestations outside the nervous system}

Children with NF1 are predisposed to myoproliferative disease, to bone abnormalities, and to vascular abnormalities.

Kevin Shannon (UCSF) presented a talk on the effects of MEK inhibitors in mouse models of acute myeloid leukemia and myeloproliferative neoplasms, including juvenile myelomonocytic leukemia. Interestingly, his data suggest that Raf/MEK/ERK signaling plays a central role in the pathologic growth of hematopoietic tumors that have inactivated NF1. However, he found that there are disease stage-specific effects of treatment with MEK inhibitors in that advanced cancers such as AML initiated by NF1 inactivation are highly dependent on Raf/MEK/ERK signaling and sensitive to MEK inhibitors [11]. By contrast, early stage neoplasms in which NF1 inactivation is the dominant (or only) cell autonomous genetic lesions are less "addicted" to Raf/MEK/ERK signaling, but respond to MEK inhibition by modulating aberrant growth. Importantly, this treatment does not eradicate mutant cells. These data have implications for treating benign neoplasms and fully malignant cancers in persons with NF1.

David Ingram (Indiana University School of Medicine) addressed inflammation in the adventitia of the vessel wall and identified a central role for macrophages. It is notable that the recruitment of bone marrow macrophages takes months. Potential treatment regimens to interrupt recruitment of Nfl heterozygous macrophages to inflamed neointima were addressed. Switching topics to assess the possibility that dysregulated Ras signaling could lead to aneurismal vessel disease, Dr. Ingram presented mouse models based on ApoE receptor signaling that when 
provided a high lipid or high cholesterol diet developed thickened adventitia and elevated oxidative stress. This oxidative stress has been seen in an $N f 1$ drosophila model, and provides potential in treatment paths for aneurismal vessel disease. Likewise, the ROS pathway is now a target for novel therapeutic intervention.

Kimberly Jett (University of British Columbia, Canada) discussed the effect of vitamin D3 treatment on bone density (BMD) in NF1 patients. They investigated the therapeutic potential of high dose oral vitamin D3 on bone mineral density in NF1 patients with vitamin D3 deficiency. The dose that maintained the serum 25-hydroxyvitamin D3 level $>30 \mu \mathrm{g} / \mathrm{l}$ was used. Treated subjects had significantly improved BMD in hip and lumbar spine in comparison to untreated subjects. Further studies are needed to elucidate the mechanisms responsible for reduced bone mineral density in NF1.

\section{Cautionary notes}

The NF community is focused on using preclinical studies to guide clinical trials.

Matthias Karajannis (NYU Langone Medical Center) discussed how preclinical trials are useful to inform clinical trials by reviewing the use of cultured primary tumor cells, immortalized/transformed cells, and presumptive cancer stem cells. While useful for cell-based high throughput screens, the challenges associated with using these in vitro models were highlighted, including the development of secondary genetic or epigenetic changes that may arise in culture, as well as the inability to assess tumor-host and host-tumor interactions. As an alternative, he discussed advantages of genetically engineered mouse models for preclinical studies.

Scott Plotkin (Massachusetts General Hospital) highlighted the difficulties in directly comparing the preclinical mouse model to human patients. Variable expressivity of NF1 and NF2, effects of other genes, gene/ environment interactions are limits to the ability of mouse models to inform human disease. Perhaps, the greatest limitation of the mouse models is their inability to model the impact of disease on quality of life that is decreased in NF1, NF2 and schwannomatosis patients. Understanding how psychological and social factors influence the experience of living with NF will require human rather than animal studies.

\section{Update: neurofibromatosis type 2}

The NF2 patients are predisposed to vestibular schwannomas, meningiomas and intramedullary tumors. A large part of the basic science talks was centered continuing improvement of understanding of NF2 role in tumor initiation and fine tuning of mouse models of different tumor types that allow preclinical therapeutics.

NF2 models + mechanisms

Filippo Giancotti (Memorial-Sloan Kettering Cancer Hospital) summarized recent advances in our understanding of the function of the NF2 gene product Merlin. He outlined potential models for Merlin's tumor suppressor function. He noted that, in addition to playing a role at or near the cell cortex, Merlin also enters into the nucleus to repress the E3 ubiquitin ligase CRL4-DCAF1 [12]. In addition, he presented evidence that Merlin's entry into the nucleus and interaction with DCAF1 can repress activation of YAP independently of the canonical Hippo pathway described in Drosophila.

Chunling Yi from Joseph Kissil's lab (The Wistar Institute) identified Angiomotin as a novel binding partner for Merlin, establishing a physical link between Merlin and the tight junction-associated Crumbs apical polarity complex [24]. Her data illustrated that Merlin functions through Angiomotin to control Rac-Pak signaling and also implicated Angiomotin as an integral component of Hippo signaling. Through the use of an orthotopic model of NF2, she demonstrated that Angiomotin is required for tumorigenesis stemmed from loss of Nf2, suggesting that Angiomotin should be considered a potential therapeutic target for NF2.

Julian Boggiano from Richard Fehon's lab (The University of Chicago) identified the Sterile 20 kinase Tao- 1 as an additional upstream regulator of the Hippo pathway from an in vivo RNAi screen in Drosophila. Tao-1, like other HSW pathway genes, is required to maintain proper organ size during development and several HSW pathway target genes are upregulated in Tao-1 RNAi tissue. In addition, Tao-1 can directly phosphorylate Hippo at a critical regulatory site, providing a clear mechanism for Hippo activation. Interestingly, Tao-1 has recently been implicated as a negative regulator of microtubule stability, suggesting a possible link between HSW signaling and microtubule dynamics.

Duojia Pan (Johns Hopkins University) presented mouse genetic studies on the functional link between Merlin and the Hippo pathway. Building on their recent demonstration that the Merlin-deficient phenotypes of multiple tissues can be suppressed by heterozygous deletion of YAP [25], he presented evidence that attenuation of TEAD, the DNAbinding partner of the YAP coactivator, also suppresses Merlin-deficient phenotypes in transgenic mouse models. These findings provide additional support for an evolutionarily conserved role of Merlin in regulating YAP from Drosophila to mammals. 
Georg Halder (MD Anderson Cancer Center) is using Drosophila as a model system to study the Hippo tumor suppressor pathway. He and his colleagues have previously shown that Merlin as well as the Fat cadherin and the crumbs receptor act as inputs into the core Hippo pathway [8]. They have now used a genome-wide RNAi screen in cultured cells and identified the Capping proteins A and B, known to restrict F-actin polymerization, as regulators of the Hippo pathway. Interestingly, they found that modulating F-actin organization by knock-down of Capping proteins or by overexpression of the formin Diaphanous in vivo caused severe over proliferation by hyperactivation of the Hippo pathway effector Yorkie. These results suggest that the actin cytoskeleton may transduce mechanical forces into the Hippo pathway.

Michel Kalamarides (Hopital Beaujon, Inserm U674, France) presented new insights mouse models of meningioma. He has previously shown that biallelic loss of $N f 2$ is rate-limiting for meningioma development with mice developing grade I meningiomas. Recently, he identified a prostaglandin D synthase-positive (PGDS) meningeal precursor as the cell of origin for murine meningioma, and showed that $N f 2$ inactivation in these cells before the formation of the three meningeal layers accounts for the heterogeneity of the meningiomas [10]. He presented a new technology based on the RCAS/tv-a system that he is currently using to characterize the PGDS cell as a meningioma progenitor cell. Finally, he presented a new mouse model of grade II and III meningioma by combining $p 16^{\text {Ink4a }} ; p 15^{\text {Ink4b }} ; p 19^{\text {Arf }}$ inactivation to $N f 2$ loss restricted to arachnoid cells thus mimicking human meningioma tumorigenesis.

Using mouse models, in which $N f 2$ inactivation was specifically induced in mammary gland luminal epithelium at different stages of development, Jie Huang (Ohio State University) showed that Merlin is critically involved in breast development and physiological adjustment during pregnancy. Inactivation of $N f 2$ from mammary epithelium during early development leads to substantial under development of mammary buds at E12.5. Inactivation during mid-to-late pregnancy decreases cell proliferation and induces apoptosis leading to decreased alveolar morphogenesis, decreased milk production and malnourishment of the offspring. Inactivation of $\mathrm{Nf} 2$ during early gestation also inhibits cell proliferation and causes nursing problems during first parturition. Cellular defects caused by $N f 2$ inactivation included alterations in the Jak2/Stat5 signaling pathway and defective modulation in tight junction components as the epithelium underwent transition from pregnancy to lactation. Interestingly, after multiple pregnancies, the $N f 2$ defective mice develop myoepithelioma-like breast tumors at high frequency.
Matthieu Peyre (Hopital Beaujon, Inserm U674, France) presented mouse models for $N f 2$-meningiomas. Data from array analysis of human tumors and mouse models point to different genetic alterations in the initiation and progression phases of meningiomas. A conditional mouse model, in which Merlin was specifically deleted in arachnoidal cells showed meningothelial proliferation and formation of meningiomas in $20 \%$ of mice at 12 months. If additionally the entire Cdkn2ab locus was inactivated ( $p 19^{\text {Arf }}$ is floxed, $p 15^{\text {Ink } 4 \mathrm{~b}}$ carries an insertion mutation and $p 16^{\text {Ink4a }}$ a point mutation) the mean survival decreased to 3.5 months and $69 \%$ of the mice developed meningiomas. Most tumors were grade I, but also higher grade tumors (grades II and III) were observed with frequent brain invasion, which the authors monitored with elegant confocal techniques. Meningiomas from these mice did not reveal gross chromosomal abnormalities different to the ones found in NF2 patients. This mouse model with short latency for tumor development and the diversity of grades makes this model an invaluable tool for investigating new treatments for meningiomas.

Sarah Burns (Ohio State University) summarized the published data on pAKT activation in Merlin-deficient tumors and then presented data on the use of two compounds, AR42 (a histone deacetylase [HDAC] inhibitor) and AR12 (an AKT inhibitor). She showed an effect of AR42 in meningioma and schwannoma cells in vitro. As a mouse model they used xenotransplants with a luciferaseexpressing, Merlin-deficient meningioma cell line BenMen-1. AR12 treatment led to a reduction of tumor growth of $42 \%$ after 1 month and $65 \%$ after 3 months. AR42 led to tumor reduction by $86 \%$ after 1 month and $94 \%$ after 3 months. When treatment with AR42 was stopped, there was minimal re-growth.

The last talk was by Wei Li (Memorial-Sloan Kettering Cancer Hospital). He presented data on how the E3 ubiquitin ligase CRL4 (DCAF1) connects to an important pathway in tumorigenesis of Merlin deficient tumors, i.e. the hippo pathway. The authors looked at contact inhibition in keratinocytes and showed that under confluent condition one finds more phosphorylation of YAP, the transcription co-activatior downstream of Hippo/Mst 1/2. Further, YAP phosphorylation is increased in confluent cells as is phosphorylation of Mst 1/2. The down-stream effector Lats $1 / 2$ can be seen in the nucleus under confluent conditions. However, in renal carcinoma cells which do not contain Salvador, a binding partner of Mst 1/2, Merlin can still induce YAP phosphorylation, and thus does not need Salvador. He concluded that Merlin can direct YAP phosphorylation via DCAF1. This is supported by experiments using another cell line meso 33, which is deficient of Merlin. In these cells re-introduction of Merlin can induce YAP, which can be inhibited by DCAF1. 


\section{NF2 clinical research}

The clinical session on NF2 was extremely well attended and showed the groundbreaking clinical/translational research now ongoing for patients with this condition.

Gareth Evans (St Mary's Hospital, Manchester, UK) reviewed the topic of gliomas in NF2. Although astrocytoma forms a constituent of the NF2 diagnostic criteria, the main predisposition in NF2 is actually to grade $1 / 2$ ependymomas which show a predilection for the cervical spine but can involve other spinal areas and medulla. Occasional low grade astrocytomas occur intracranially in NF2 but not high grade. EMA immunohistochemistry and electron microscopy should be used in difficult cases to distinguish between ependymomas and astrocytomas At the molecular level, although NF2 mutations have been described in a proportion of sporadic spinal ependymomas they have not been found in several large series of high grade gliomas. In summary, although gliomas are part of NF2, the type associated is ependymomas and not astrocytomas. The group suggested that NF2 diagnostic criteria should be revised to include ependymoma.

Jaishri Blakeley (Johns Hopkins University) presented early results from the Phase 0 study of anti- EGFR/ErbB2 therapy in sporadic and NF2-related human vestibular schwannomas (VS) (NCT00863122). Adult patients with VS were prescribed lapatinib $1,500 \mathrm{mg}$ daily orally for 10 days immediately preceding surgery for VS resection. Overall, lapatinib was well tolerated and no dose modifications were necessary due to adverse events. Tumor pharmacokinetic data showed good tumor penetration and immunohistochemical analysis confirmed expression of EGFR and/or ErB2 in the majority of case and control samples. The data are promising and support continued testing of lapatinib as a potential therapy for human VS.

Fabio Nunes (Massachusetts General Hospital) then presented an update on the MGH series of NF2 patients treated with bevacizumab ( $5 \mathrm{mg} / \mathrm{kg}$ i.v. every 2 weeks) this time concentrating on responses in meningioma, a recent paper having highlighted meningioma response to bevacizumab in one patient [7]. The MGH group looked at their series of 14 patients with evaluable meningiomas (NCT01207687). An initial radiographic response was noted in 12/40 meningiomas $(30 \%)$. Over the duration of the study, only seven tumors $(17.5 \%)$ remained in response, 20 progressed $(50 \%)$, and 13 remained stable $(32.5 \%)$. The results suggest that bevacizumab was only effective in decreasing meningioma size in a minority of meningiomas. There was a presentation from Simone Ardern-Holmes (The Children's Hospital at Westmead, Australia) on the potential value of Positron Emission Tomography with Gallium-Octreotide to estimate somatostatin receptor density in order to select NF2 patients most likely to respond to somatostatin analogs. The Sydney based group had performed Gallium-Octreotide PET scans on 12 NF2 patients. Preliminary results indicated that octreotide avidity varies considerably among NF2 associated tumors, with meningiomas showing higher responses. A young patient had recently been commenced on the somatostatin analog octreotide (Sandostatin LAR), with a further patient about to begin treatment. Clinical response as indicated by symptomatic improvement and 3 dimensional volumetric MRI data will be followed.

There followed two papers looking at quality of life. Rachael Hornigold (Guy's and St. Thomas' NHS Foundation Trust, UK) presented her new NF2 measure the NFTI-QOL which was specifically developed to assess a disease-specific quality of life score for NF2. The SF-36 and EuroQOL were used for validation. NFTI-QOL scores correlated strongly and significantly with the EuroQOL and with all domains of the SF-36 (Pearson correlation, $p \leq 0.001$. THE NFTI-QOL appears to be a promising new QOL measure specific to NF2 that is easy to administrate and score (only 8 items). Vanessa Merker (Massachusetts General Hospital) presented her research trying to correlate QOL with whole body tumor burden in patients with NF1, NF2 and schwannomatosis. Tumor volume and number were correlated with SF-36 subscales. The study included 142 subjects with NF1, 53 subjects with NF2, and 49 subjects with schwannomatosis. While tumor volume and count did not correlate with any SF-36 scales in patients with NF1 or NF2, tumor volume was positively correlated with bodily pain in schwannomatosis.

Finally, Amanda Bergner (Johns Hopkins Comprehensive Neurofibromatosis Center) presented an update on the Schwannomatosis International Database, a collaborative effort to allow for pooling of data from multiple centers. The database was designed to be a platform from which multiple research endeavors can be launched. The data points were chosen specifically to provide adequate information to identify patients appropriate for a variety of research studies while remaining de-identified to protect patient confidentiality. A steering committee composed of experts in the fields of schwannomatosis has been established, the database has been designed and built, an effective and efficient infrastructure for coordinating this project has been established, and applications for use of the data in clinical trials are now being received. The project had increased communication and collaboration within the schwannomatosis community of experts.

A panel session focused on long term hearing preservation strategy in NF2 patients. Michel Kalamarides (Hopital Beaujon, France) presented his experience in conservative (watch and wait) approach for selected cases. He showed that spontaneous hearing preservation is 
frequent in NF2 patients ( $81 \%$ at 5 years in this series) indicating that a tailored treatment should be based on the knowledge of the natural history of each vestibular schwannoma (VS) in each patient. Michael Link (Mayo Clinic) shared his experience of radiosurgery in NF2 patients. He has treated 32 VS with a 7-year follow-up. Hearing preservation was rare (23\%) compared to sporadic VS. However, $80 \%$ of irradiated VS showed no growth allowing cochlear implantation in case of positive responses during electric stimulation. This strategy could provide long-term auditory rehabilitation with open-set speech discrimination in some cases ( 2 out 4 ). This strategy needs further confirmation. Derald Brackmann (House Ear Clinic) presented his large experience in the proactive treatment of smaller tumors allowing hearing preservation rates nearly comparable to those obtained in patients with sporadic tumors $(60 \%)$. However, there is a tendency for growth of new schwannomas within the surgical field $(60 \%)$. He presented an original surgical strategy performed in 45 NF2 patients: middle fossa decompression of vestibular schwannoma in only hearing ear. The average period of hearing maintenance was 2.1 years. The shortterm hearing preservation rate was approximately $90 \%$. The 1-year hearing preservation rate was 63\% [20]. Understanding the duration of hearing preservation can enable more effective counseling of patients considering middle cranial fossa decompression for VS. Scott Plotkin (Massachusetts General Hospital) presented an update (median time for treatment: 14 months $(3-42 \mathrm{~m}$.) of his series of patients treated with bevacizumab, an anti-VEGF monoclonal antibody well tolerated in NF2 (NCT012 07687) [17]. Twenty-three patients were eligible for a hearing response evaluation: 12 had a hearing response (defined as a significant increase in the word-recognition score, as compared with baseline), 8 had stable hearing, and 3 had progressive hearing loss. The median time to response was 3 months and the median time to hearing loss is superior to 3 years.

In conclusion, the NF community is unceasingly concentrated on identifying the mechanisms of tumor development in NF1 and NF2. Lagging behind are understanding of schwannomatosis, cognitive changes, and bone abnormalities in NF1. Continual improvement of mouse models to provide platforms for validating new anticancer drugs, performing preclinical trials to inform ongoing clinical trials, and designing optimally new clinical studies including combination therapy remain top priorities in NF research.

Open Access This article is distributed under the terms of the Creative Commons Attribution Noncommercial License which permits any noncommercial use, distribution, and reproduction in any medium, provided the original author(s) and source are credited.

\section{References}

1. Acosta MT, Kardel PG, Walsh KS, Rosenbaum K, Gioia GA, Packer RJ (2011) Lovastatin improves cognitive deficits in NF1: safety and efficacy study. Ped Neurol 45(4):241-245

2. Bar EE, Lin A, Tihan T, Burger PC, Eberhart CG (2008) Frequent gains at chromosome $7 \mathrm{q} 34$ involving BRAF in pilocytic astrocytoma. J Neuropathol Exp Neurol 67(9):878-887

3. Brown JA, Emnett RJ, White CR et al (2010) Reduced striatal dopamine underlies the attention system dysfunction in neurofibromatosis-1 mutant mice. Hum Mol Genet 19:4515-4528

4. Cancer Genome Atlas Research Network (2008) Comprehensive genomic characterization defines human glioblastoma genes and core pathways. Nature 455:1061-1068

5. Denayer E, Ahmed T, Brems H et al (2008) Spred1 is required for synaptic plasticity and hippocampus-dependent learning. J Neurosci 28:14443-14449

6. De Raedt T, Walton Z, Yecies JL, Li D, Chen Y, Malone CF, Maertens O, Jeong SM, Bronson RT, Lebleu V, Kalluri R, Normant E, Haigis MC, Manning BD, Wong KK, Macleod KF, Cichowski K (2011) Exploiting cancer cell vulnerabilities to develop a combination therapy for ras-driven tumors. Cancer Cell 20(3):400-413

7. Goutagny S, Raymond E, Sterkers O, Colombani JM, Kalamarides $M$ (2011) Radiographic regression of cranial meningioma in a NF2 patient treated by bevacizumab. Ann Oncol 22:990-991

8. Halder G, Johnson RL (2011) Hippo signaling: growth control and beyond. Development 138:9-22

9. Hyman SL, Gill DS, Shores EA, Steinberg A, North KN (2007) T2 hyperintensities in children with neurofibromatosis type 1 and their relationship to cognitive functioning. J Neurol Neurosurg Psychiatry 78(10): 1088-1091

10. Kalamarides M, Stemmer-Rachamimov AO, Niwa-Kawakita M et al (2011) Identification of a progenitor cell of origin capable of generating diverse meningioma histological subtypes. Oncogene 30:2333-2344

11. Lauchle JO, Kim D, Le DT et al (2009) Response and resistance to MEK inhibition in leukaemias initiated by hyperactive Ras. Nature 461:411-414

12. Li W, You L, Cooper J et al (2010) Merlin/NF2 suppresses tumorigenesis by inhibiting the E3 ubiquitin ligase CRL4 (DCAF1) in the nucleus. Cell 140:477-490

13. Liu C, Sage JC, Miller MR et al (2011) Mosaic analysis with double markers reveals tumor cell of origin in glioma. Cell 146:209-221

14. Lopez G, Torres K, Lev D (2011) Autophagy blockade enhances HDAC inhibitors' pro-apoptotic effects: potential implications for the treatment of a therapeutic-resistant malignancy. Autophagy 7(4):440-441 (Epub 2011 Apr 1)

15. Lorenzo J, Barton B, Acosta MT, North KN (2011) The mental motor and language development of toddlers with neurofibromatosis type 1. J Pediatr 158:660-665

16. Orr BA, Bai H, Odia Y, Jain D, Anders RA, Eberhart CG (2011) Yes-associated protein 1 is widely expressed in human brain tumors and promotes glioblastoma growth. J Neuropathol Exp Neurol 70:568-577

17. Plotkin SR, Stemmer-Rachamimov AO, Barker FG 2nd et al (2009) Hearing improvement after bevacizumab in patients with neurofibromatosis type 2. N Engl J Med 361:358-367

18. Pride N, Payne JM, Webster R, Shores EA, Rae C, North KN (2010) Corpus callosum morphology and its relationship to cognitive function in neurofibromatosis type 1 . J Child Neurol 25:834-841

19. Raabe EH, Lim KS, Kim JM et al (2011) BRAF activation induces transformation and then senescence in human neural stem 
cells: a pilocytic astrocytoma model. Clin Cancer Res 17:3590-3599

20. Slattery WH, Hoa M, Bonne N et al (2011) Middle fossa decompression for hearing preservation: a review of institutional results and indications. Otol Neurotol 32:1017-1024

21. Verhaak RG, Hoadley KA, Purdom E et al (2010) Integrated genomic analysis identifies clinically relevant subtypes of glioblastoma characterized by abnormalities in PDGFRA, IDH1, EGFR, and NF1. Cancer Cell 17:98-110

22. Wu J, Dombi E, Jousma E, Scott Dunn R, Lindquist D, Schnell BM, Kim MO, Kim A, Widemann BC, Cripe TP, Ratner N (2011) Preclincial testing of Sorafenib and RAD001 in the $\mathrm{Nf}$ (flox/flox) ;DhhCre mouse model of plexiform neurofibroma using magnetic resonance imaging. Pediatr Blood Cancer. doi: $10.1002 /$ pbc. 23015

23. Yang et al (2008) Nf1-dependent tumors require a microenvironment containing $\mathrm{Nf} 1 \pm$ and c-kit-dependent bone marrow. Cell 135(3):437-448

24. Yi C, Troutman S, Fera D et al (2011) A tight junction-associated Merlin-angiomotin complex mediates Merlin's regulation of mitogenic signaling and tumor suppressive functions. Cancer Cell 19:527-540

25. Zhang N, Bai H, David KK et al (2010) The Merlin/NF2 tumor suppressor functions through the YAP oncoprotein to regulate tissue homeostasis in mammals. Dev Cell 19:27-38 